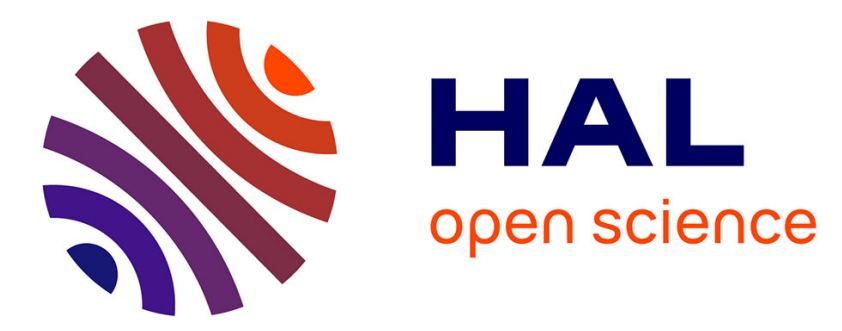

\title{
Céphalées chroniques quotidiennes et effet de la prise en charge médicale. Une étude longitudinale
}

Margaux Bouteloup, Rose-Angélique Belot, Magalie Bonnet, André Mariage, Fabrice Vuillier

\section{- To cite this version:}

Margaux Bouteloup, Rose-Angélique Belot, Magalie Bonnet, André Mariage, Fabrice Vuillier. Céphalées chroniques quotidiennes et effet de la prise en charge médicale. Une étude longitudinale. Psychologie clinique et projective, 2020, n²8 (2), pp.37. 10.3917/pcp.028.0037 . hal-03578771

\section{HAL Id: hal-03578771 \\ https://hal.science/hal-03578771}

Submitted on 28 Feb 2022

HAL is a multi-disciplinary open access archive for the deposit and dissemination of scientific research documents, whether they are published or not. The documents may come from teaching and research institutions in France or abroad, or from public or private research centers.
L'archive ouverte pluridisciplinaire HAL, est destinée au dépôt et à la diffusion de documents scientifiques de niveau recherche, publiés ou non, émanant des établissements d'enseignement et de recherche français ou étrangers, des laboratoires publics ou privés. 


\section{Auteurs :}

M. Bouteloup ${ }^{1}$

R.-A. Belot ${ }^{2}$

M. Bonnet ${ }^{3}$
A. Mariage 4
F. Vuillier ${ }^{5}$

Titre : Céphalées chroniques quotidiennes et effet de la prise en charge médicale. Etude longitudinale de l'évolution psychique d'un sujet au Rorschach

Correspondance : Margaux Bouteloup, 87 rue des granges, 25000 BESANCON, 06.36.92.43.97

Courriel : margaux.bouteloup@live.fr

\footnotetext{
${ }^{1}$ Psychologue clinicienne - Docteure en psychologie - UFR SLHS Bourgogne/Franche-Comté Laboratoire de Psychologie EA3188

${ }^{2}$ Maître de conférences en psychologie clinique - UFR SLHS Bourgogne/Franche-Comté Laboratoire de Psychologie EA3188 : rose-angélique.belot@ univ-fcomte.fr

${ }^{3}$ Maître de conférences en psychologie clinique - UFR SLHS Bourgogne/Franche-Comté Laboratoire de Psychologie EA3188 : magalie.bonnet@univ-fcomte.fr

${ }^{4}$ Professeur de Psychologie clinique - UFR SLHS Bourgogne/Franche-Comté - Laboratoire de Psychologie EA3188 : andré.mariage@univ-fcomte.fr

${ }^{5}$ Professeur Universitaire Praticien Hospitalier Neurologie Anatomie - UFR SMP

Bourgogne/Franche-Comté, Laboratoire de neurosciences EA481 : fabrice.vuillier@univ-fcomte.fr
} 


\title{
Céphalées chroniques quotidiennes et effet de la prise en charge médicale.
}

\author{
Etude longitudinale de l'évolution psychique d'un sujet au Rorschach
}

\begin{abstract}
RESUME : Les céphalées chroniques quotidiennes (CCQ) touchent environ 3\% de la population française et sont particulièrement difficiles à prendre en charge. Cet article, à partir d'une étude de cas, interroge l'évolution psychique d'une patiente présentant des CCQ. Des entretiens de recherche et deux Rorschach à 6 mois d'intervalle ont été réalisés (avant et après la prise en charge médicale). Une très nette amélioration au niveau de la symptomatologie a été constatée. Toutefois, les résultats au Rorschach et les entretiens de recherche mettent en évidence des mouvements de déstabilisation après la disparition de la symptomatologie. La migraine semble comporter une fonction importante dans l'économie psychosomatique du sujet.
\end{abstract}

MOTS-CLES : Migraine ; céphalées chroniques quotidiennes ; Rorschach ; économie psychosomatique ; étude de cas.

KEYWORDS Migraine, daily chronic headache, Rorschach, psychosomatic, clinical case

\section{INTRODUCTION}

\section{Les céphalées chroniques quotidiennes : quelques chiffres}

Les céphalées chroniques quotidiennes touchent environ 3\% de la population française avec une surreprésentation de 3 femmes pour 1 homme. Au sens neurologique strict, les céphalées chroniques quotidiennes appartiennent à la catégorie des céphalées primaires, c'est-à-dire des douleurs de la région crânienne sans lien avec une lésion ou anomalie identifiable, au même titre que les migraines et les céphalées (appelées céphalées de tension). Les migraines se caractérisent par des crises à répétition, d'une durée de $4 \mathrm{~h}$ à $72 \mathrm{~h}$, se manifestant essentiellement par de pénibles et douloureux maux de tête accompagnés de nausées. Elles nécessitent une réduction, voire un arrêt total, de toute activité et s'accompagnent souvent d'un besoin de se coucher dans le noir à l'abri de tout stimulus. Les céphalées de tension sont moins intenses, plus diffuses et très courantes. Les céphalées chroniques quotidiennes, quant à elles, correspondent à un tableau clinique de maux de tête pendant au moins 15 jours par mois durant 3 mois. Il s'agit souvent d'une «complication» de la symptomatologie migraineuse et sont la plupart du temps le résultat d'un glissement entre l'utilisation préventive de médicaments et la surconsommation de ces derniers par le patient (plus de 10 analgésiques par mois), qui présente au départ un « simple » trouble migraineux.

L'étude GRIM2005 (Lantéri-Minet et al., 2007) a rapporté que seulement 30.8\% des patients présentant des migraines étaient activement inscrits dans un système de soins. Chez les $70 \%$ restant, $40.8 \%$ des sujets pratiquent l'auto-médication. Or, le risque principal de l'auto-médication est la surconsommation, pouvant entraîner comme effet secondaire des céphalées dites de rebond. Ainsi, une spirale peut très rapidement s'installer. Le patient a alors besoin d'un médicament pour calmer ses maux de tête. Malheureusement, la symptomatologie réapparaîtra dès la fin d'action du traitement, nécessitant une nouvelle prise de médicament. Il est très fréquent que les patients ne se rendent pas compte de cette escalade chimiothérapique. Ils constatent seulement que les traitements sont de moins en moins efficaces. C'est le plus souvent au cours de la consultation avec le neurologue que la prise de conscience a lieu. Afin d'endiguer le processus, l'hospitalisation pour sevrage est très fortement recommandée. Cette proposition peut représenter pour le patient soit un soulagement d'être pris en charge, soit un rejet catégorique, le sujet se sentant assimilé à un toxicomane. 
L'étude des céphalées sous le prisme de la psychologie n'est pas récente. Pour Marty, les céphalées font partie des somatisations fonctionnelles régressives non-évolutives. Selon lui, les migraines constitueraient des «mécanismes secondaires de défense vis-à-vis de l'irruption à la conscience d'un ensemble conflictuel xedipien dont au moins certains éléments représentables ont été d'abord refoulés » (Marty, 1976). Plus généralement, Marty (1951) décrit la migraine comme une inhibition douloureuse de l'acte de penser, conférant à cette symptomatologie une valeur économique dans le fonctionnement psychosomatique du sujet. Par ailleurs, des capacités de mentalisation incertaines et un attachement insécure (Rossi et al., 2005 ; Savi et al., 2005) ont été repérés comme facteur de risque psychosomatique pour le patient migraineux (Bouteloup, Belot, Bonnet, Mariage, \& Vuillier, 2017). Rueff-Escoubès (2008) propose, quant à elle, d'envisager la migraine comme un «liant fixateur » du fonctionnement psychique du sujet et une protection de la réalité par le repli : en ce sens, la symptomatologie peut devenir indispensable à la stabilité psychosomatique. Marty (1951) s'inscrit également dans cette conception, envisageant même pour certains sujets la migraine comme un rempart contre une menace d'effondrement psychotique.

Sur un plan plus quantitatif, des études ont montré que la migraine était associée à la dépression, à l'anxiété (Baldacci et al., 2015 ; Ligthart, Gerrits, Boomsma, \& Penninx, 2013) mais aussi au stress (Fève, 2008; Huber \& Henrich, 2003). De plus, les patients présentant des céphalées auraient des caractéristiques psychologiques significativement différentes de la population générale, à savoir qu'ils seraient plus embarrassés, irritables, pessimistes et présenteraient davantage de difficulté à se relaxer (Pradalier, Mavre, Servière, Vincent, \& Dry, 1990 ; Huber \& Henrich, 2003).

Des études ont également mis en avant qu'il existe des différences significatives entre les caractéristiques psychologiques des patients présentant des migraines et ceux souffrant de céphalées chroniques quotidiennes (Zampieri, Tognola, \& Galego, 2014). Ces derniers présenteraient davantage de troubles anxieux et dépressifs, mais également des idées suicidaires, une perte d'espoir et une altération de la concentration.

En outre, selon le type de migraine que le patient présente, épisodiques ou chroniques, il existe des différences significatives dans la faculté du sujet à décrire ses émotions. Pour Galli et al., l'alexithymie peut représenter un facteur de risque dans la chronicisation des migraines (Galli et al., 2017). Enfin, et comme pour les céphalées, des variables psychologiques ont été repérées comme associés à la chronicité des migraines (Radat et al., 2009) : une baisse de la qualité de vie du sujet, un degré de stress psychologique élevé, l'utilisation de certains mécanismes de défense de catastrophisation et d'évitement comme stratégie de coping et enfin l'utilisation majoritaire d'un locus de contrôle externe (attribution d'une causalité externe aux évènements subis).

La dernière étude avec la méthodologie Rorschach auprès de patients migraineux date du début des années 2000 en Italie (Pizza, Spitaleri, \& Colucci d'Amato, 2001). En France, en 1951, Marty a proposé avec Fain le test de Rorschach (Marty, 1951). Toutefois, Marty écrit que les résultats n’ont montré aucune particularité par rapport à l'investigation clinique réalisée au préalable. C'est à Timsit (1972, 1978), associé à Boulanger (Boulanger, Timsit, 1974), que nous devons plusieurs travaux portant sur l'étude des patients migraineux au Rorschach. Plusieurs signes liés selon ces auteurs à différentes carences sont présents dans les protocoles : un nombre de réponses réduit, évoquant une carence de la vie fantasmatique; peu de kinesthésies humaines, traduisant une carence des relations objectales; un nombre de réponses banales augmenté, signant une carence des sens; une augmentation du pourcentage de réponses formelles de bonne qualité, révélant une carence de la pensée. Il met également en évidence des distorsions kinesthésiques très importantes au Rorschach pour les sujets céphalalgiques (Timsit, 1978). Quelques années plus tard, Timsit et al. (1987) relèvent 
un profil caractéristique des patients migraineux et se retrouvant dans plus de la moitié des cas. Ce profil associe une forte activité pulsionnelle et un processus de contrôle et de contention : il qualifie ce profil de répressif. L'activité pulsionnelle se traduit par une prévalence de réponses déterminées par la couleur, par le TRI et par le choc couleur aux planches II et III. Les processus de contrôle s'apprécient à travers un F+\% élevé, témoin de l'exactitude de la pensée et des distorsions des réponses kinesthésiques. Les autres profils retrouvés sont d'allure anxio-dépressive (27\% des cas) ou relevant de modalités de conversion hystérique ou pré-psychotiques (16\% des cas). Ces résultats nécessitent aujourd'hui d'être réactualisés et approfondis.

\section{METHODOLOGIE}

Notre équipe, constituée de neurologues, psychologues et méthodologistes, a conduit une recherche longitudinale auprès de patients migraineux dans un service hospitalier de neurologie. Toutes les autorisations réglementaires ont été obtenues ${ }^{6}$. Tous les participants signent un formulaire de consentement éclairé avant le début de l'inclusion.

La recherche est proposée à tous les patients consultant pour la première fois pour des céphalées primaires, âgés entre 25 et 60 ans, sans antécédent psychiatrique et par ailleurs bien portants. Il leur est proposé, entre autres, la passation du Rorschach à l'entrée dans le service, avant qu'une prise en charge médicale ne soit proposée. Une seconde passation a lieu 6 mois plus tard, une fois le traitement médicamenteux mis en place et bien toléré. Un entretien de recherche est également mené à l'entrée dans le protocole et 6 mois après.

Notre objectif ici est de réussir à mieux cerner, grâce à l'étude de cas, les éléments qui composent l'économie psychosomatique du sujet. En effet, ce modèle défend l'idée que tout sujet peut avoir recours à des régulations d'appoint, passant parfois par une symptomatologie physique, pour assurer une homéostasie psychique et psychosomatique : l'appréciation du fonctionnement psychosomatique est particulièrement riche avec les méthodologies projectives (Debray, 1983 ; Pheulpin, 2014). Pour les patients souffrant de céphalées chroniques quotidiennes, nous avons pu constater que plusieurs sujets présentaient des similarités de fonctionnement, parfaitement reflétées par l'étude de cas et les protocoles Rorschach de Myriam que nous proposons.

\section{SITUATION CLINIQUE ${ }^{7}$}

Myriam, 40 ans, est mariée et a 4 enfants âgés de 4 à 18 ans. Elle travaille dans le secteur sanitaire et social. Actuellement, Myriam vit dans un contexte difficile, marqué par d'importantes contraintes et difficultés familiales. Ses parents sont issus de l'immigration. Myriam est née et a toujours vécu en France. Elle retourne une fois par an avec beaucoup de plaisir dans le pays d'origine de ses parents. Elle est d'un abord souriant et agréable, ponctuant très régulièrement son discours d'éclats de voix, d'humour et de rires, rendant le tout très vivant. Elle parle abondamment, de façon directe et utilise parfois des mots vulgaires. Myriam raconte son histoire avec un certain détachement mais fait ressentir au chercheur qui l'écoute l'intensité du bouillonnement interne qui l'habite. Par ailleurs, elle génère rapidement chez les professionnels une certaine inquiétude quant à l'évolution de ses troubles et leur sédation.

\section{Situation somatique à l'entrée dans le protocole}

\footnotetext{
${ }^{6}$ Comité d'éthique, $\mathrm{n}^{\circ} \mathrm{CNIL}$, enregistrement dans Clinical Trials.

${ }^{7}$ Certaines données ont été modifiées pour préserver l'anonymat de la patiente.
} 
Myriam dit présenter des migraines depuis son enfance. Elle consulte aujourd'hui en service hospitalier de neurologie car depuis 2 ans, la symptomatologie a évolué : les migraines sont quasiconstantes, avec une intensité allant de 5 à 10. La fréquence des migraines dépend, d'après Myriam, de son état psychique : "quand je suis très énervée, stressée, contrariée, mais bon... comme je suis comme ça tout le temps, et ben j'ai tout le temps mal (rire). Ou un conflit, ou... j'arrive pas à gérer, et ça se transforme en mal de tête ».

A l'issue de la première rencontre avec le neurologue, le diagnostic de céphalées chroniques quotidiennes par abus médicamenteux est posé. Le neurologue propose donc une hospitalisation de 5 jours à Myriam pour un sevrage.

A la perspective d'une nette amélioration de la symptomatologie évoquée par le neurologue après cette hospitalisation, Myriam s'inquiète de savoir si elle aura encore des migraines après : "oui parce qu'autant là, c'est ingérable comme ça, tout le temps, j'en peux plus, mais j'aimerais bien en garder une ou deux, car au moins quand j'ai mal à la tête, on me laisse tranquille».

Par ailleurs, Myriam a du cholestérol. De plus, depuis quelques années, lorsque Myriam s'énerve, des plaques d'eczéma apparaissent sur les avant-bras et au niveau du cou.

\section{Enfance de Myriam}

Myriam est issue d'une grande fratrie dans une famille à recompositions multiples. Myriam décrit ses relations avec sa mère comme étant de très mauvaise qualité. Sa mère est, selon ses propos, «rejetante, manipulatrice et avec beaucoup d'emprise », et ce encore aujourd'hui. Myriam a cependant pu compter sur une belle-mère aimante. Le père de Myriam était très souvent absent, mais elle le décrit comme une personne soutenante, avec qui les relations étaient très satisfaisantes.

A l'école, Myriam n'avait que peu d'amis. Elle a passé son adolescence à jouer au foot avec les garçons de son quartier. Dans ce contexte familial et amical, Myriam dit ne s'être jamais laissé marcher sur les pieds : "j'étais quelqu'un qui répondait beaucoup, qui avait du répondant, mais qu'on n'écoutait jamais, alors je me suis réfugiée dans le sport. Ou bien je faisais le ménage pour me défouler ».

\section{$\underline{\text { Relations familiales actuelles }}$}

Il y a quelques années et en un mois, Myriam a perdu plusieurs membres de sa famille, dont son père et sa belle-mère. Myriam est prise dans un maillage culturel, du fait de l'origine de ses parents, qu'elle qualifie de «malsain et hypocrite». Elle pense ne pouvoir compter sur personne en cas de difficultés. Pour Myriam, le poids culturel l'oblige à s'occuper de sa mère, qui vit seule, et à lui rendre visite régulièrement, mais elle n'y trouve aucun plaisir. Elle devait même par le passé prendre un anxiolytique avant chaque visite pour supporter les réflexions maternelles blessantes dont elle fait constamment l'objet. Pour Myriam, sa mère, tout comme le reste de sa famille, "piétine dans sa vie». Tout son entourage la sollicite en sachant qu'elle ne sait pas dire non : «Je suis la conchita moi, j'encaisse tout ». Myriam s'est toujours sentie rejetée : «j'ai l'impression qu'on se fout de ma gueule, on m'a toujours pris pour une moins que rien ».

Le mari de Myriam a les mêmes origines qu'elle. Elle le décrit comme très soutenant pour l'éducation des enfants et le quotidien. Ses principes culturels le poussent à encourager Myriam à s'occuper de sa mère et de sa famille, créant parfois des conflits dans le couple. Le couple reste malgré tout solide et uni face aux intrusions et aux provocations de la mère de Myriam.

Myriam décrit la relation avec ses enfants comme un bonheur, bien que celle-ci soit conflictuelle, notamment avec les deux aînés, adolescents. Elle se trouve parfois peu tolérante et dépassée : «Moi, il m'arrive des fois de crier sur mes enfants, et j'ai des flashs je me dis "oh purée je croirais entendre ma mère ». Et là je... je dis ohlala non pas ça!». La perspective de se retrouver avec tous 
ses enfants à la maison la panique car elle sait qu'il risque d'y avoir des conflits : «alors qu'est-ce que je fais? Je prends mon sac et je me casse. C'est le seul moyen. Et après je rentre quand leur père rentre. Pour avoir la paix, j'esquive ma maison ».

\section{Gestion du conflit}

Myriam nous explique être très impulsive : «Je gueule. Je pique ma crise, je les insulte. Et je me tais plus, je parle tout le temps, je m'arrête pas, et je rabâche, je rabâche. Ça se termine, je suis épave avec une grosse fatigue et une grosse douleur à la tête, et puis voilà ce que j'ai gagné (rire). On dirait que je suis passée sous un train ».

Myriam explique qu'elle ne sait pas dire les choses calmement : "je sais pas faire, c'est ça mon tort, c'est-à-dire que moi je vais supporter pendant des mois, des années, et puis un truc et je vais monter en flèche. Une soupape catastrophique. Et aucun regret, aucun remords, rien à foutre. Mais après, je culpabilise, pas d'avoir dit ce que je pense mais d'en être arrivée là. J'ai un très gros problème avec la culpabilité, parce qu'on m'a toujours culpabilisée depuis gamine (rire) ». Myriam utilise l'image d'un citron pressé pour se décrire. Elle a l'impression que personne ne l'écoute lorsqu'elle évoque son épuisement: "J'ai tout fait, j'ai bien montré que ça n'allait pas mais bon, ça les a pas dérangés».

\section{Activités}

Myriam se décrit comme très active, «sur pile », générant tout à la fois l'admiration, les moqueries et le stress de son entourage: "Je piétine toute la journée, j'ai toujours à faire. Je m'invente du boulot, ou il faut que j'ai l'esprit occupé j'en sais rien. On dirait que j'ai besoin de cette montée d'adrénaline et de faire plein de choses à la fois, mais du coup je stresse et je stresse tout le monde. Ça fait tellement d'années que je vis avec cette pression, elle fait partie de moi, si ça se trouve j'en ai peut-être besoin. Même si je lâche prise je veux l'avoir ». A l'évocation de cette possibilité de lâcher-prise, le chercheur évoque les tâches ménagères qui pourraient peut-être s'assouplir : "Mais je n'en ai pas le droit, parce qu'il faut que tout soit rangé comme il faut, parce que j'ai décidé comme ça. Il faut que ça marche comme moi je veux, sinon je pète les plombs. Ohlala, j'ai mal à la tête ça y est, ça lance ». Elle répètera souvent durant l'entretien : "peut-être que je cherche la perfection, mais il faut que ce soit fait en temps et en ordre ».

\section{$\underline{\text { Symptomatologie migraineuse à la sortie du protocole } 6 \text { mois plus tard }}$}

Lorsque nous rencontrons Myriam 6 mois plus tard, la symptomatologie migraineuse s'est nettement améliorée : l'hospitalisation a permis d'enrayer le processus indésirable de sur-consommation de médicaments et d'instaurer un traitement de fond qui fonctionne très bien, puisque Myriam n'a fait que 4 ou 5 crises en 7 mois. Elle se dit satisfaite, mais regrette que le traitement lui ait fait prendre un peu de poids (il s'agit d'un effet secondaire « normal » du traitement de fond). Elle évoque l'hospitalisation comme plus difficile qu'elle ne l'aurait pensé, car sa famille lui a manqué.

Au niveau de son organisation familiale, rien n'a évolué, mais Myriam en parle avec un détachement encore plus prononcé que lors des premières rencontres, suscitant de nouveau de l'inquiétude chez le chercheur pour Myriam et ses enfants : «moi je m'en fous, ils font ce qu'ils veulent et moi je fais ce que j'ai à faire et basta. Même mes gosses maintenant, j'ai lâché l'affaire, ils me parlent mal et tout, moi j'aurais jamais parlé comme ça à mon père, et même à ma mère alors que j'ai de quoi, mais c'est pas mon problème, ils verront bien plus tard, ils se débrouillent. J'ai appris à lâcher prise. » 


\section{PRESENTATION ET ANALYSE DES RESULTATS AU RORSCHACH}

\section{Les indices du psychogramme}

\section{Table 1}

\begin{tabular}{ccc} 
T1 & Normes & T2 \\
\hline $\mathrm{R}=20$ & $\mathrm{R}=28.16$ & $\mathrm{R}=18$ \\
$\Sigma \mathrm{K}=1$ & $\Sigma \mathrm{K}=2.42$ & $\Sigma \mathrm{K}=0$ \\
$\Sigma \mathrm{k}=2$ & $\Sigma \mathrm{k}=3.82$ & $\Sigma \mathrm{k}=3$ \\
$\Sigma \mathrm{C}=2$ & $\Sigma \mathrm{C}=3.36$ & $\Sigma \mathrm{C}=1$ \\
$\Sigma \mathrm{E}=0.5$ & $\Sigma \mathrm{E}=1.04$ & $\Sigma \mathrm{E}=0$ \\
$\mathrm{RC} \%=45 \%$ & $\mathrm{RC} \%=35.94 \%$ & $\mathrm{RC} \%=44 \%$ \\
\hline
\end{tabular}

Les indices ci-dessus du psychogramme aux deux temps de la recherche (T1 et T2) s'éloignent tous des normes (De Tychey, Huckel, Rivat, Claudon, 2012). L'indice R traduit une faiblesse représentative face au contenu projectif et interroge sur la qualité des processus de pensée. De même, la faiblesse des réponses kinesthésiques nous questionne sur les capacités de mentalisation de Myriam et la souplesse de son fonctionnement psychique.

Le $\mathrm{RC} \%$, supérieur à la norme, montre une certaine réceptivité affective, mais Myriam semble ne pas pouvoir intégrer ces motions pulsionnelles dans son fonctionnement économique. En effet, la faiblesse du $\mathrm{C}$ indique des difficultés à traiter l'affect sollicité et plus encore, à le lier à une représentation $(\mathrm{K})$, reflétant des difficultés d'élaboration psychique.

Au deuxième temps, le fonctionnement psychique de Myriam d'un point de vue économique semble être encore davantage ralenti, puisque le nombre de réponses et les indices témoignant d'une liaison représentation/affect sont encore plus inférieurs aux normes qu'au temps 1. Les processus de pensée apparaissent comme davantage figés et les capacités de symbolisation défaillantes.

Table 2

\begin{tabular}{ccc} 
T1 & Normes & T2 \\
\hline $\mathrm{F} \%=60 \%$ & $\mathrm{~F} \%=57.81 \%$ & $\mathrm{~F} \%=56 \%$ \\
$\mathrm{~F} \%$ élargi $=100 \%$ & - & $\mathrm{F} \%$ élargi $=100 \%$ \\
$\mathrm{~F}+\%=42 \%$ & $\mathrm{~F}+\%=60.86 \%$ & $\mathrm{~F}+\%=30 \%$ \\
$\mathrm{~F}+\%$ élargi $=60 \%$ & - & $\mathrm{F}+\%$ élargi $=56 \%$ \\
$10 \mathrm{~B} / 4 \mathrm{P}^{8}$ & $2 \mathrm{~B} / 1 \mathrm{P}$ & $9 \mathrm{~B} / 2 \mathrm{P}$ \\
\hline
\end{tabular}

Le recours à la forme se situe dans la norme aux deux temps $(\mathrm{F} \%$ : $\mathrm{T} 1=60 \%, \mathrm{~T} 2=56 \%)$, témoignant d'une certaine capacité chez Myriam à s'adapter à la réalité extérieure. Cela signe également l'absence d'une effraction de l'enveloppe psychique, et ce, aux deux de temps de notre rencontre. L'indice $\mathrm{B} / \mathrm{P}$ de Fisher et Cleveland va d'ailleurs dans le sens d'une enveloppe surinvestie, signe d'un Moi-Peau carapace (Anzieu, 1985), accentué au T2. L'augmentation des réponses $\mathrm{B}$ par rapport aux réponses $\mathrm{P}$ traduit un renforcement défensif des frontières du Moi et de façon certaine, une modification de l'économie psychosomatique.

\footnotetext{
${ }^{8}$ Le coefficient de référence pour l'indice B/P est de 2 . AU T1, il est de 2,5 et au T2, il est de 4,5.
} 
Cependant, la faiblesse importante du F+\%, non compensé par le F+\% élargi, nous interroge sur la qualité du rapport au réel et les capacités de socialisation de Myriam. Au T2, le F+\% chute encore davantage, signant, à notre sens, un épuisement du Moi, dont nous discuterons la signification plus loin.

Table 3

\begin{tabular}{ccc} 
T1 & Normes & T2 \\
\hline $\mathrm{G} \%=45 \%$ & $\mathrm{G} \%=36.83 \%$ & $\mathrm{G} \%=72 \%$ \\
$\mathrm{D} \%=45 \%$ & $\mathrm{D} \%=57.24 \%$ & $\mathrm{D} \%=6 \%$ \\
$\mathrm{Dd} \%=5 \%$ & $\mathrm{Dd} \%=3.13 \%$ & $\mathrm{Dd} \%=11 \%$ \\
\hline
\end{tabular}

Au T1, Myriam utilise un mode d'appréhension mixte, alternant entre des perceptions globales et plus détaillées : ces résultats permettent de relever des possibilités de mécanismes d'isolation pour traiter l'excitation. Par contre, au T2, le D\% s'effondre et est très inférieur aux normes au profit d'un $\mathrm{G} \%$ très élevé. Ceci peut traduire une réaction passive et une appréhension simpliste des planches : serait-ce pour diminuer l'impact de la situation projective, lutter contre les émergences internes ? L'augmentation du Dd\% peut appuyer cette interprétation, signant le besoin de réduire aux petits détails les sollicitations pour pouvoir fonctionner au plan psychique et produire une réponse. Toutefois, les Dd apparaissent dans des localisations inhabituelles et interrogent les capacités de conformité et de socialisation de Myriam.

\section{Table 4}

\begin{tabular}{ccc} 
T1 & Normes & T2 \\
\hline IA $\%=5 \%$ & IA $\%=13.3 \%$ & IA $\%=5 \%$ \\
\hline
\end{tabular}

Le IA\% est très inférieur à la norme mais stable à T1 et T2. Pourtant, Myriam semble sensible à la sollicitation latente de certaines planches et reconnaît l'aspect angoissant via les commentaires (Pl. I T1 : «j'aimerais pas le croiser », Pl. X T1 : «olala, j'aime pas... », Pl. X T2 : « ces deux trucs ils me font peur »). Myriam fonctionne essentiellement par la décharge pulsionnelle dans les commentaires abondants de l'aspect angoissant, mais ne réussit pas à traduire les aspects anxiogènes dans les réponses et leurs contenus.

\section{Table 5}

\begin{tabular}{ccc} 
T1 & Normes & T2 \\
\hline $\mathrm{A} \%=52 \%$ & $\mathrm{~A} \%=42.55 \%$ & $\mathrm{~A} \%=45 \%$ \\
$\mathrm{H} \%=5 \%$ & $\mathrm{H} \%=15.85 \%$ & $\mathrm{H} \%=5 \%$ \\
$\mathrm{Obj}=5$ & - & $\mathrm{Obj}=7$ \\
$\mathrm{Scène}=1$ & - & $\mathrm{Scène}=3$ \\
$\mathrm{Ban}=6$ & 4.83 & Ban $=4$ \\
\hline
\end{tabular}

Malgré la faiblesse du nombre de réponses et de kinesthésies, la présence de plusieurs réponses Scène, renforcée au T2, vient témoigner d'une certaine capacité d'activité psychique, voire d'élaboration. De même, le nombre de Ban et le A\% supérieurs aux normes nous permettent de relativiser les difficultés de socialisation mises en avant avec l'analyse du $\mathrm{F}+\%$, très faible, et du $\mathrm{Dd} \%$. Ces deux indices témoignent de capacités certaines d'insertion dans le monde environnant, et 
ce, sans tomber dans une pensée stéréotypée. Cependant, le $\mathrm{H} \%$ très faible aux deux temps et l'augmentation des réponses Objet et du A\% peuvent traduire un mouvement de déshumanisation voire de dévitalisation. Les contenus des réponses, que nous reprendrons plus loin, appuient cette interprétation.

Sensibilité à la sollicitation latente des planches

\section{Table 6}

\section{T1}

Pl. III : «y a des formes qui prêtent à confusion»

$\mathrm{Pl}$ IV : «On va dire une espèce de monstre qui arrive avec ses gros panards [...]. Mais c'est pas beau à voir.»

Pl. VII : « une grenouille » (évoque au T2 que c'était sa mère la grenouille)

Pl. IX : «un pot de fleur», « un vase»

Utilise souvent le terme « imaginer »
T2

$\mathrm{Pl}$. VI : "C'est celle-là que j'avais imaginé ma mère comme une grenouille. Mais là je vois un monstre [...] et qui est moche ».

$\mathrm{Pl}$. VII : «deux petits chiots qui sont sur un rocher en bas et qui se regardent»

Pl. IX : «fontaine », «vase », « support»

Utilise souvent le terme « imaginer»

L'utilisation à de nombreuses reprises du terme «imaginer» montre que Myriam a intégré la consigne projective et est capable de fonctionner sur un plan imaginaire. De plus, Myriam est sensible à la sollicitation latente de certaines planches à T1 comme à T2

\section{Déshumanisation et dévitalisation des réponses}

\section{Table 7}

T1

Pl. III : « on peut dire que c'est deux personnes qui ont été dessinées de façon un peu bizarroïdes autour d'une table ronde. On peut pas dire que c'est une femme ou un homme, bon là c'est une femme parce que $c$ 'est des talons aiguilles, mais y a des formes qui prêtent à confusion. Ou des chiens ».

$\mathrm{Pl}$. V : papillon en phase terminale

Pl. VI : hésitation entre une peau et un tapis Pl. VIII : «deux ours qui sont en train de grimper [...] là leur verdure » Pl. IX : un pot de fleur ou un vase
T2

$\mathrm{Pl}$ III : "celle là je m'en rappelle, on dirait deux chiens assis l'un en face de l'autre »

Pl. V : papillon étalé

Pl. VI : hésitation entre un tapis ou un animal «désossé » ou «arraché sa peau »

Pl. VIII : «Oh bah oui je m'en souviens, c'était deux ours qui montaient un rocher, je les vois toujours. Je n'ai plus envie de dire que c'est des rochers, $j$ 'ai plus envie de dire que c'est un tapis avec des dessins d'ours. »

Pl. IX : « une fontaine, [...] une statue en forme de lampe décorée bizarrement par un artiste. » 
Alors que la réponse «deux personnes dessinées » montrait un scénario relationnel de bonne qualité avec valorisation de la féminité par le détail phallique, au T2, la représentation «deux chiens assis l'un en face de l'autre » s'inscrit dans un registre narcissique. De plus, la difficulté autour de l'identification sexuelle à la planche III est évacuée au T1 par un mouvement de déshumanisation, qui se confirme au T2. De même, la dévitalisation aux pl. V, VI, VIII et IX est prégnante et s'intensifie entre le T1 et le T2. Le gel pulsionnel apparaît au T2 avec des animaux qui deviennent un décor de tapis et une réponse fontaine qui devient statue.

\section{Représentation de soi}

\section{Table 8}

Pl. I : "Une chauve souris, enfin je crois. [...] Elle est pas belle, j'aimerais pas la croiser » $\mathrm{Pl} . \mathrm{V}:$ «Oh, un petit papillon qui a des ailes bien larges. Bon $i l^{9}$ aurait pu mettre des couleurs quand même parce que là il fait pitié. On va dire qu'il est en phase terminale, il est pas beau ».

Pl. VI : «Un tapis. [...] Il est moche, pour lui donner une belle image, on va dire que c'est une peau de bête posée, douce. »
Pl. I : «J'imaginais une grenouille je me souviens, mais en fait ça veut rien dire. Une chauve souris qui est pas belle. »

$\mathrm{Pl} . \mathrm{V}$ : «Un espèce de papillon bizarre étalé, de grandes ailes, mais qui est pas beau non plus. On sait pas trop si on peut l'imaginer papillon ou chauve souris, non papillon pas beau plutôt ».

Pl. VI : «un tapis, une peau d'animal qu'on a fait en tapis. Je trouve qu'il ressemble à rien à part un tapis qui est mal fini là. »

Enquête : "Un tapis, un animal qu'on a désossé, arraché sa peau».

La représentation de soi apparaît négative et très dévalorisée, avec l'évocation de contenus «moches » ou «pas beaux » aux deux temps.

A la planche VI, Myriam essaye de contre-investir cette dévalorisation au T1 en donnant une «belle image » au tapis, mais au T2, c'est un «tapis mal fini » qui est évoqué, nous faisant penser à un Moi qui s'effiloche, s'use et à une attaque au plan du narcissisme. Les ailes larges peuvent traduire le besoin de tenir le coup face au poids sur les épaules : Myriam endosse tout, mais le corps, lui, ne tient plus. Des angoisses mortifères d'annihilation apparaissent au T1 avec l'évocation d'une « phase terminale », qui semble se concrétiser au T2 : le papillon est étalé, terrassé, comme à terre.

Par ailleurs « une peau de bête posée, douce » au T1 devient «un tapis, un animal qu'on a désossé, arraché sa peau ». Ici les difficultés liées à une enveloppe trouée, déchiquetée apparaissent de façon explicite, en contradiction avec l'indice Barrière/Pénétration étudié précédemment.

\footnotetext{
${ }^{9}$ Fait référence à « l'artiste » qui aurait conçu les planches
} 
Image maternelle et relations précoces

\section{Table 9}

T1

Pl. II : « un diamant posé sur un truc entouré»

Pl. IX : « un pot de fleur qui a été mal dessiné [...], un vase tout coloré, tout beau, enfin qui croit qu'il est beau mais je mettrais pas ça chez moi »

Pl. VII : «on peut imaginer que c'est une grenouille assez grosse puisqu'on voit ses pattes. Ou un crapaud je sais pas. Bien grosse, bien grasse? [...] c'est moche hein?»

Pl. X : «j'en ai assez vu. Franchement on aurait dit n'importe quoi. »
T2

Pl. II : « un diamant posé sur un support» Pl. IX : « on dirait une fontaine. Une lampe. Pour moi elle m'inspire rien du tout. On dirait un espèce de vase décoré bizarrement. Une statue en forme de lampe décorée bizarrement. On peut imaginer le support $\gg$.

$\mathrm{Pl}$ IV : «Olala, celle là je me rappelle. C'est celle-là que j'avais imaginé ma mère comme une grenouille».

Pl. X : «ces deux trucs ils me font peur »

Myriam semble rechercher une relation de soutien dans une dimension narcissique, mais la dévalorisation des contenus aux pl. VII et IX traduisent des relations précoces de mauvaise qualité, ayant entraîné un narcissisme fragile et des difficultés à solliciter l'aide d'autrui.

La grenouille devenant crapaud évoque une imago maternelle dévalorisée mais aussi phallique : elle est d'ailleurs évoquée à la pl. IV au T2 et non plus à la pl. VII. L'aspect gros et gras de la grenouille évoque un envahissement de cette imago maternelle, envahissement qui se traduit bien au T2 puisque Myriam se rappelle qu'elle a évoqué une image de sa mère à la planche IV., planche liée à un percept massif, imposant. Au T1, Myriam se défend de la séparation en prenant les devants sur un mode caractériel : $\mathrm{Pl}$. X «j'en ai assez vu. Franchement on aurait dit n'importe quoi ». Cette séparation est davantage élaborée sur le plan psychique au T2, avec l'expression d'un affect de peur, signant des possibilités de réorganisation psychique.

Expression de l'agressivité

Table 10

$\mathrm{T} 1$

Réponses pinces ou pincettes aux planches

I, IV et VI.
$\mathrm{T} 2$

Réponses pinces ou pincettes aux planches I et IV.

Pl. III : « deux chiens $[. .$.$] en train de se battre un$ os, et que le meilleur gagne »

L'agressivité ne s'exprime que sur des contenus «pinces » et «pincettes » et toute conflictualisation au T1 semble évincée par les commentaires de mise à distance ou de dévalorisation du matériel. Au T2, ce mode d'expression minimisé est toujours présent, avec cependant une possibilité de 
conflictualisation des relations qui apparaît à la planche III, mais au prix d'un glissement de contenu (d'une personne, elle évoquera ensuite uniquement des chiens). On remarque également le jaillissement d'une représentation pulsionnelle massive mais subie à l'enquête à la planche VI au T2 (cf table 8). Ces éléments traduisent des capacités de réorganisation psychique, même si de nombreux indices semblent montrer que les difficultés d'élaboration psychique sont plus importantes au T2.

\section{Commentaires et critiques du matériel}

\section{Table 11}

$\mathrm{T} 1$

Pl. I : «j'aimerais pas le croiser»

Pl. II : «Je kiffe les diamants, mais j'en n'ai jamais eu, j'aurais dî changer de mari ». «Pour moi ça veut rien dire »

Pl. VI : «Vous voulez que je vous dise quoi ? » Enquête : "pauvre bête»

$\mathrm{Pl}$. VII : «Vous avez déjà vu des grenouilles bien grosses? C'est moche hein?»

$\mathrm{Pl}$. IX : «mal dessiné [...] le design est pas

top. [...] Je mettrai pas ça chez moi »

PL. X : «Olala, j'aime pas...»

"Quand on regarde après on a de drôles

d'images devant les yeux»

«Je crois que j'en ai assez vu. Franchement

on aurait dit n'importe quoi ».
$\mathrm{T} 2$

Pl. I : «en fait ça veut rien dire »

$\mathrm{Pl}$. II : « maintenant je sais que ça veut rien dire en fait ce truc »

Pl. III : «j'aimerais pas croiser ça un soir»

Enquête pl. VII : "les formes sont marrantes mais sans plus, mais comme j'ai imaginé, c'est marrant »

Contenu «pas beau » ou «moche »; pl. I, pl. V; p. VI, pl. IX ; pl. X

Contenu «moche $»$ : pl. IV, pl. VI, pl. VII

Myriam agrémente ses réponses de nombreux commentaires et critiques du matériel. Ceux-ci traduisent des mécanismes de défense de type caractériel et comportemental. Face à la sollicitation des planches et à l'afflux d'excitations qu'elles provoquent, ce registre défensif est majeur. Ce mode de fonctionnement est présent au $\mathrm{T} 1$ et au T2, bien que moins marqué à la deuxième passation.

\section{DISCUSSION GENERALE}

\section{Le fonctionnement psychique de Myriam}

A travers l'analyse de l'anamnèse de Myriam, un fonctionnement psychique de type névrose de caractère semble se dessiner (selon la classification psychosomatique de Pierre Marty). En effet, Myriam explique qu'en cas de conflit, ce sont bien ses dispositions caractérielles qui s'expriment : elle crie, insulte, rabâche, jusqu'à ce qu'un mal de tête s'installe et stoppe le processus. Nous comprenons donc aisément que Myriam s'inquiète de la prise en charge proposée par le neurologue, qui vise à réduire considérablement la symptomatologie migraineuse, et donc à modifier son fonctionnement sur un mode économique. 
De plus, les modalités de relation à autrui ne semblent exister pour Myriam que sur un mode conflictuel ou caractériel : elle est en conflit permanent avec sa famille, ses enfants, mais aussi avec elle-même.

Par ailleurs, le recours au comportement semble également une modalité économique privilégiée pour Myriam, et ce, depuis son adolescence. En effet, Myriam a toujours utilisé le ménage ou le sport pour «se défouler ». Aujourd'hui encore, l'activité tient une place prépondérante, avec une dimension de contrôle importante puisque Myriam souhaite que les choses soient faites selon son idée, signe d'un travail bien fait. Myriam s'impose beaucoup d'actions et une façon de faire rigide, mais semble peu à peu perdre tout plaisir à cuisiner, prendre soin d'elle, activités pourtant ressourçantes auparavant. A la perspective de l'hospitalisation, Myriam appréhende de n'avoir aucune tâche quotidienne à réaliser. Nous percevons donc que dans l'économie psychosomatique de Myriam, le comportement tient également une place importante, au même titre que le caractère.

\section{Modalités de traitement de l'excitation au Rorschach}

Ces dimensions caractérielles apparaissent également au Rorschach: Myriam est sensible à la sollicitation latente des planches, mais cherche à évacuer l'excitation provoquée par le percept via des commentaires nombreux: la décharge verbale tient une place importante dans les deux protocoles. La faiblesse du IA\% traduit également ce fonctionnement par la décharge. Myriam évacue par les commentaires l'angoisse sans qu'elle puisse l'inscrire sur le plan psychique. Ces commentaires semblent donc venir prendre le relai face à des difficultés manifestes d'élaboration psychique. Nous nous interrogeons cependant sur l'efficacité de ces mécanismes défensifs, Myriam développant depuis plusieurs années diverses somatisations ajoutées à l'aggravation de ses migraines (eczéma, cholestérol...).

Les dimensions conflictuelles et agressives apparaissent à certaines planches très contenues dans les réponses, et à d'autres elles apparaissent sous la forme de représentation pulsionnelle intense. Myriam illustre ce fonctionnement au quotidien dans sa gestion du conflit : elle passe de la rétention de tout ce qu'elle pense à une explosion incontrôlée. Ce mode d'expression nous interroge sur la possibilité pour Myriam de gérer l'alternance entre les pôles actif et passif.

Par ailleurs, Myriam exprime, aussi bien dans son discours qu'à travers le Rorschach, l'envahissement que représente pour elle ses fonctions maternelles et familiales. Personne dans son entourage ne semble pouvoir lui venir en aide, notamment avec sa mère, avec qui les relations sont décrites comme de mauvaise qualité. Myriam est à la recherche d'un soutien, d'un support, qu'elle trouve chez le neurologue, qui lui propose de l'hospitaliser et de «prendre soin d'elle » durant 5 jours. Mais le fonctionnement psychique de Myriam lui permet-il de lâcher-prise pendant une semaine ? Quelle place la symptomatologie migraineuse a-t-elle dans son fonctionnement et plus largement dans son économie psychosomatique?

\section{$\underline{\text { L'évolution de Myriam traduite au Rorschach : la place de la symptomatologie migraineuse }}$}

Le premier constat marquant de cette double passation est la confirmation de l'inquiétude du chercheur à travers l'analyse des psychogrammes. En effet, au T1, les résultats de Myriam sont plutôt dans les normes, bien que quelques indices nous interrogent déjà sur les difficultés d'élaboration psychique de notre patiente, tels que l'augmentation du nombre de réponses Détails $(\mathrm{D} \%)$, de réponses humaines $(\mathrm{H} \%)$, la faiblesse de la qualité formelle des réponses $(\mathrm{F}+\%)$, la quasiabsence de réponses $\mathrm{K}$ et $\mathrm{C}$. A T2, le psychogramme révèle un écart significatif des normes sur la 
grande majorité des indices, dont le $\mathrm{G} \%$, le $\mathrm{R}$ et le $\mathrm{F}+\%$, ce dernier étant extrêmement bas. Or, le $\mathrm{F}+\%$ est un indicateur élémentaire de l'adaptation au réel mais aussi de la force du Moi et de la solidité de la structuration psychique. Ceci rejoint les constatations de Rueff-Escoubès, Marty, et plus généralement de Dejours, évoquant la migraine comme un liant fixateur de la structure ou un rempart contre une menace d'effondrement psychique voire psychotique. La présentation de cette situation clinique montre, comme chez d'autres sujets rencontrés, une déstabilisation de l'économie psychosomatique du fait de l'amélioration des CCQ. En effet, la nette amélioration de la symptomatologie grâce à la prise en charge médicale de Myriam est venue renforcer la défaillance d'un Moi fragile. L'analyse qualitative des réponses et la comparaison entre T1 et T2 illustre cette usure du Moi.

L'étude de la représentation de soi à $\mathrm{T} 1$ et $\mathrm{T} 2$ révèle également une faille narcissique importante qui se renforce dans le temps, Myriam semblant avoir désormais une image d'elle-même négative.

Cependant, dans une lignée plus positive, l'apparition d'une possibilité de conflictualisation des relations au $\mathrm{T} 2$ perceptible à la réponse de la planche III, absente au T1, nous encourage à penser que la disparition de la symptomatologie migraineuse oblige Myriam à débuter un travail psychique. Ainsi, l'aggravation des indices du psychogramme au T2 pourrait aussi s'envisager comme le signe d'une déstructuration ou d'un affaiblissement des capacités du moi précédant un mouvement de restructuration de l'appareil psychique. En effet, pour Dejours (2009) notamment, les somatisations peuvent être symbolisantes, traçant le chemin d'une réorganisation.

L'analyse des Rorschach et du discours de Myriam nous conduisent à postuler que l'hospitalisation pour le sevrage médicamenteux était nécessaire pour endiguer les céphalées chroniques quotidiennes, mais nécessite également un accompagnement au long cours afin d'appréhender la dimension économique que peut revêtir la symptomatologie. En effet, il apparaît pour Myriam que la migraine peut être une expression somatique qui vient en lieu et place d'un travail de mentalisation et d'élaboration, notamment des modalités relationnelles et conflictuelles. Elle dira d'ailleurs dès l'annonce du sevrage son souhait de "garder certains maux de tête » car ils lui permettent des moments de tranquillité. Les migraines semblent jouer un rôle important dans son équilibre psychosomatique et peuvent constituer une symptomatologie d'appoint qui lui permet de traiter les excitations non liées et de répondre de façon économique à ses mécanismes de défense caractériels et comportementaux.

\section{EVOLUTION ET PERSPECTIVES}

A l'occasion de la réalisation de ce travail d'analyse, nous avons consulté le dossier médical de Myriam pour reprendre l'ensemble des informations de la symptomatologie migraineuse. A la lecture, nous avons constaté que, malheureusement, nos inquiétudes se sont avérées fondées: quelques mois après la sortie du protocole de recherche, Myriam a arrêté son traitement. La symptomatologie s'est de nouveau chronicisée de manière journalière. Le rapport du médecin indique que Myriam «est toujours aussi active. Elle a bien conscience de faire beaucoup trop de choses et de la nécessité de lever un peu le pied».

Aussi, à l'issue de cette étude de cas, il apparaît nécessaire de penser des modalités de prise en charge spécifique pour ces patients migraineux fonctionnant sur un mode économique similaire à Myriam. Le travail psychothérapique à long terme apparaît difficile pour les patients présentant des névroses de caractère ou de comportement. Myriam a d'ailleurs refusé notre proposition de suivi par un autre psychologue. Nous pensons que, dans le cas de Myriam, une approche pluridisciplinaire basée sur la gestion du quotidien et des affects apparaît comme la meilleure option thérapeutique envisageable. En effet, si certains sujets ne sont pas encore en mesure d'élaborer leurs souffrances 
passées, d'autres thérapeutiques peuvent participer à modifier leur économie psychosomatique telles que l'ergothérapie, la sophrologie, la kinésiologie... Il s'agit de permettre au patient de mettre en place de nouvelles règles de vie, moins strictes, afin d'assouplir ses mécanismes de défense très prégnants du côté de l'activité, du comportement et du caractère. Des dispositifs de prise en charge sont donc à créer pour ces patients.

\section{REFERENCES BIBLIOGRAPHIQUES}

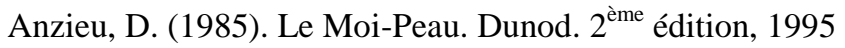

Baldacci, F., Lucchesi, C., Cafalli, M., Poletti, M., Ulivi, M., Vedovello, M., ... Gori, S. (2015). Migraine features in migraineurs with and without anxiety-depression symptoms: A hospital-based study. Clinical Neurology and Neurosurgery, 132, 74-78. https://doi.org/10.1016/j.clineuro.2015.02.017

Boulanger et timsit

Bouteloup, M., Belot, R.-A., Bonnet, M., Mariage, A., \& Vuillier, F. (2017). Migraine, qualité de l'attachement et mentalisation : étude de la dynamique psychique à partir du cas de Sylvie. Annales Médico-psychologiques, revue psychiatrique. https://doi.org/10.1016/j.amp.2017.06.007

Debray, R. (1983). Préconscient et maladie somatique. Quelques interrogations actuelles. Revue Française de Psychanalyse, (2), 527-538.

Dejours, C. (2009). Corps et psychanalyse. L'information psychiatrique, 85(3), 227-34

De Tychey, C., Huckel, C., Rivat, M., Claudon, P. (2012). Nouvelles normes adultes au test de Rorschach et évolution sociétale : quelques réflexions. Bulletin de psychologie, 521(5), 453-66. https://doi.org/10.3917/bupsy.521.0453.

Fève, A. (2008). Aspects psychologiques des migraines et des céphalées : repérages neurologiques. Revue française de psychosomatique, 34(2), 141. https://doi.org/10.3917/rfps.034.0141

Galli, F., Caputi, M., Sances, G., Vegni, E., Bottiroli, S., Nappi, G., \& Tassorelli, C. (2017). Alexithymia in chronic and episodic migraine: a comparative study. Journal of Mental Health, 26(3), 192-196. https://doi.org/10.3109/09638237.2015.1124404

Huber, D., \& Henrich, G. (2003). Personality Traits and Stress Sensitivity in Migraine Patients. Behavioral Medicine, 29(1), 4-13. https://doi.org/10.1080/08964280309596169

Lantéri-Minet, M., Massiou, H., Nachit-Ouinekh, F., Lucas, C., Pradalier, A., Radat, F., ... Hasnaoui, A. E. (2007). The GRIM2005 Study of Migraine Consultation in France I. Determinants of Consultation for Migraine Headache in France. Cephalalgia, 27(12), 1386-1397. https://doi.org/10.1111/j.1468-2982.2007.01426.x

Ligthart, L., Gerrits, M. M. J. G., Boomsma, D. I., \& Penninx, B. W. J. H. (2013). Anxiety and Depression Are Associated With Migraine and Pain in General: An Investigation of the Interrelationships. The Journal of Pain, 14(4), 363-370. https://doi.org/10.1016/j.jpain.2012.12.006

Marty, P. (1951). Aspect psychodynamique de l'étude clinique de quelques cas de céphalalgies. Revue française de psychosomatique, XVI, 3, 339-357.

Marty, P. (1976). Les mouvements individuels de vie et de mort. Paris, France : Payot \& Rivages.

Pizza, V., Spitaleri, D. L. A., \& Colucci d'Amato, C. (2001). The personality profile and alexithymic syndrome in primary headache: a Rorschach study. The Journal of Headache and Pain, 2(1), 31-37. https://doi.org/10.1007/PL00012184

Pheulpin, M. (2014). L'économie psychosomatique, une notion chère à Rosine Debray. Psychologie clinique et projective, 20(1), 167-180. doi:10.3917/pcp.020.0167

Pradalier, A., Mavre, D., Servière, R., Vincent, D., \& Dry, J. (1990). Le migraineux : recherche de caractéristiques psychologiques. La Revue de Médecine Interne.

Radat, F., Lantéri-Minet, M., Nachit-Ouinekh, F., Massiou, H., Lucas, C., Pradalier, A., ... El Hasnaoui, A. (2009). The GRIM2005 Study of Migraine Consultation in France. III: Psychological Features of Subjects With Migraine. Cephalalgia, 29(3), 338-350. https://doi.org/10.1111/j.1468-2982.2008.01718.x

Rossi, P., Di Lorenzo, G., Malpezzi, M. G., Di Lorenzo, C., Cesarino, F., Faroni, J., ... Troisi, A. (2005). Depressive symptoms and insecure attachment as predictors of disability in a clinical population of patients with episodic and chronic migraine. Headache, 45(5), 561-570. https://doi.org/10.1111/j.1526-4610.2005.05110.x

Rueff-Escoubès, C. (2008). La reine offensée. Revue française de psychosomatique, 34(2), 97. https://doi.org/10.3917/rfps.034.0097

Savi, L., Buccheri, R., Tambornini, A., De Martino, P., Albasi, C., \& Pinessi, L. (2005). Attachment styles and headache. The Journal of Headache and Pain, 6(4), 254-257. https://doi.org/10.1007/s10194-005-0200-3

Timsit 1972, 1978 


\section{Timsit et al. 1987}

Zampieri, M. A. J., Tognola, W. A., \& Galego, J. C. B. (2014). Patients with chronic headache tend to have more psychological symptoms than those with sporadic episodes of pain. Arquivos de Neuro-Psiquiatria, 72(8), 598602. https://doi.org/10.1590/0004-282X20140084 